\title{
Renal Cell Carcinoma with Double Synchronous Contralateral Adrenal Metastases
}

\author{
Masanori Ishida ${ }^{1,2}$, Kaoru Kojima ${ }^{2}$, Kuni Ohtomo' \\ Department of Radiology, ${ }^{1}$ University of Tokyo Hospital, ${ }^{2}$ Toshiba General Hospital, Tokyo, Japan
}

\begin{abstract}
A 63-year-old male patient visited our hospital with a right incidental renal tumor, which was found by ultrasonography for the follow-up study of chronic hepatitis B virus infection and diabetes mellitus. Consecutive computed tomography revealed a right renal tumor and two left adrenal tumors. Further systemic imaging study and hormonal examination suggested one right renal cell carcinoma and left adrenal metastases. We performed right nephrectomy and left adrenalectomy. The pathological diagnoses of all resected tumors were renal cell carcinoma. The patient has been in good health without any recurrence for 12 months since the operation. In patients with renal cell carcinoma, contralateral adrenal metastasis is usually associated with multiple metastases to other organs. There are a few cases of solitary and synchronous contralateral adrenal metastasis in the English literature. To our knowledge, this is the first report of a case of renal cell carcinoma with double synchronous contralateral adrenal metastases.
\end{abstract}

\section{Key Words: Adrenal glands; Neoplasm metastasis; Neoplasms; Renal cell carcinoma; Synchronous neoplasms}

This is an Open Access article distributed under the terms of the Creative Commons Attribution Non-Commercial License (http://creativecommons.org/licenses/by-nc/3.0) which permits unrestricted non-commercial use, distribution, and reproduction in any medium, provided the original work is properly cited.

\author{
Article History: \\ received 23 June, 2010 \\ accepted 1 September, 2010
}

Renal cell carcinoma (RCC) can metastasize to almost every organ, including the lungs, liver, kidneys, bones, and brain [1]. Whereas metastasis of RCC to different sites is common, contralateral adrenal metastasis is remarkably rare [2]. There are a few cases of solitary and synchronous contralateral adrenal metastasis in the English literature $[3,4]$, but there are no reports on double synchronous contralateral adrenal metastases with primary RCC. Herein, we report one such exceptional case along with a discussion of the literature.

\section{CASE REPORT}

A 63-year-old male patient was regularly followed up because of chronic hepatitis B virus infection and diabetes mellitus. His history of present illness was a right incidental renal tumor that was found by ultrasonography (US). He had no other past history or family history. Abdominal and pelvic computed tomography (CT) revealed a 50x45 mm right renal tumor with an indistinct border.
The right renal contour was smooth, and there was no extrusion by the tumor. The CT images also revealed two left adrenal tumors (20x15 mm and $15 \times 10 \mathrm{~mm})$ (Fig. 1A). By enhanced CT (dynamic study) with the use of contrast material, the right renal tumor was heterogeneously enhanced and the degree of enhancement was weaker than the normal renal parenchyma in the early phase. The tumor showed mild wash-out in the delayed phase (Fig. 2B). We also found right renal venous invasion by the tumor (Fig. 2A). The two left adrenal tumors demonstrated the same enhanced pattern as the right renal tumor (Fig. 1B and $1 \mathrm{C})$. In the present case, the differential diagnoses of the right renal tumor on the basis of the CT findings included RCC, transitional cell carcinoma, malignant lymphoma, and metastasis. In addition, the left adrenal tumors were suspected to be metastases, adenomas, or pheochromocytomas. Including hormonal examination, laboratory data were normal except for an elevated HbA1c (8.5\%) and positivity for HBs antigen. Chest CT and bone scintigraphy demonstrated no abnormal findings. No le- 

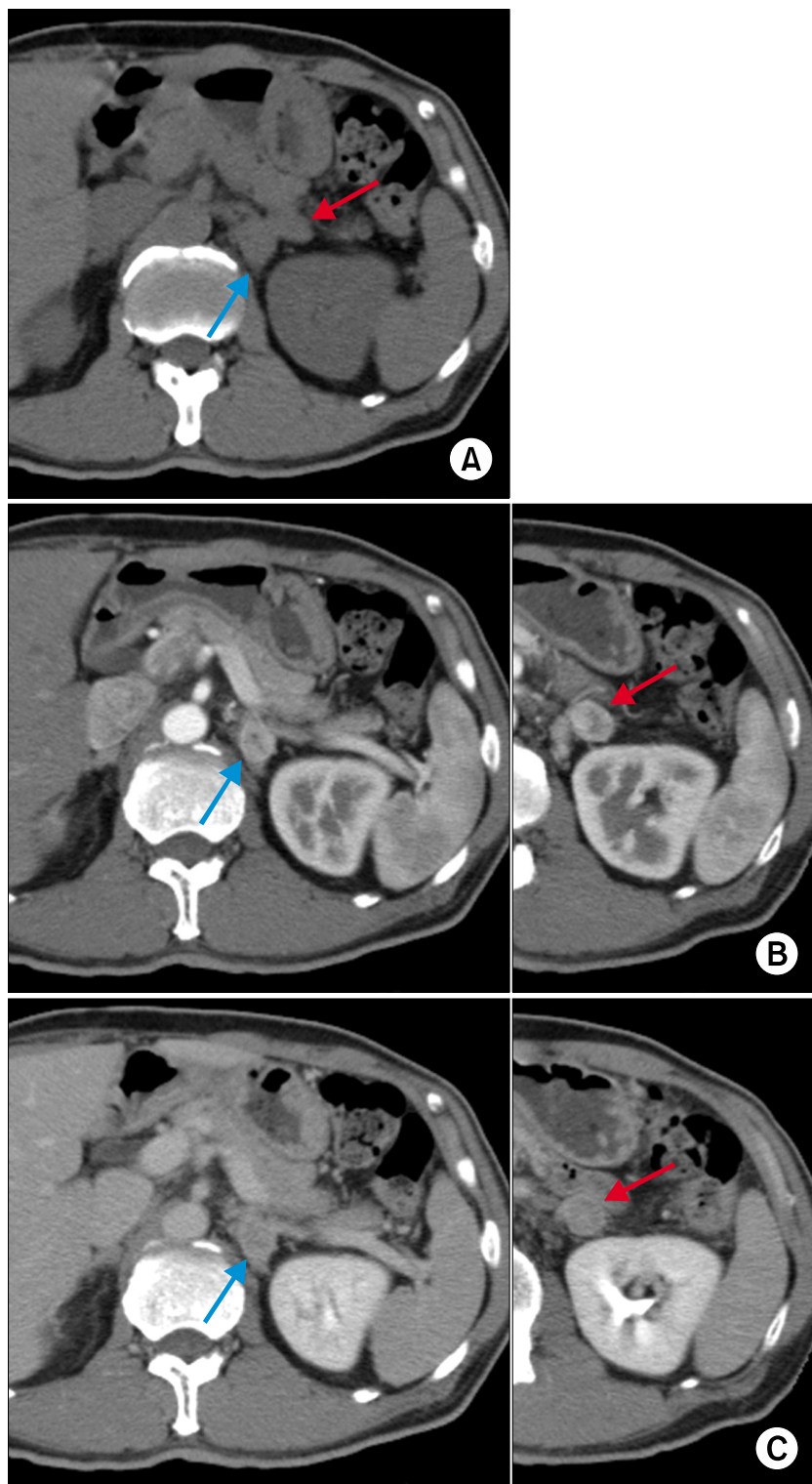

FiG. 1. Preoperative computed tomography (CT) images of left adrenal tumors (arrows). We did not identify the presence of fat in the tumors (A). The tumors showed the same dynamic contrast enhancement pattern as the right renal tumor (B, C). sions had been pointed out during the patient's past periodic checkups, including CT and US. Consequently, we clinically diagnosed right RCC and left adrenal metastases. Before the operation, ${ }^{123}$ I-MIBG scintigraphy revealed no uptake in the left adrenal region and we ruled out pheochromocytoma. Later, we performed right nephrectomy and left adrenalectomy. Macroscopically, the size of the right renal tumor was about $50 \times 45 \mathrm{~mm}$ and the sizes of the left adrenal tumors were $18 \times 15 \mathrm{~mm}$ and $15 \times 10 \mathrm{~mm}$ (Fig. 3). All were yellowish-white tumors. Pathological examination revealed that the tumor form was alveolar growth and there were many atypical cells that were composed of swelling nuclei in the clear cytoplasm. The tumor was diagnosed as RCC of the clear cell type (Fig. 4). Right renal venous invasion was also identified, but there was no lymphoid invasion. The pathological diagnosis of the resected left adrenal tumors was also RCC. Since the operation, the patient has been in good health without any recurrence for 12 months.

\section{DISCUSSION}

About $25 \%$ of patients with RCC show evidence of metastasis at the time of presentation [5,6]. The lungs, bone, lymph node, and liver are the major sites of metastatic spread [1]. It is said that the adrenal gland is the fifth organ to be metastasized [7]. Malignant involvement of the ipsilateral adrenal gland is noted in only $1.2 \%$ to $10 \%$ of cases $[8,9]$. Moreover, contralateral adrenal involvement by RCC is extremely rare. To study the frequency of metastasis in patients with RCC, Saitoh et al conducted autopsies in 1,828 patients [2]. They reported that solitary and synchronous contralateral adrenal metastases were seen in $0.19 \%$ of the patients [2]. In 2003, Lau et al described that the type of RCC that metastasized to the contralateral adrenal gland was only the clear cell type [1]. The biological mechanism of adrenal metastasis from contralateral RCC is not clear. Contralateral adrenal metastasis of RCC is thought to occur via the hematogeneous route, as is the case for other organ metastases. Some studies reported that the adrenal gland was suggested to have a higher affinity for the spread of RCC than other organs because contralateral
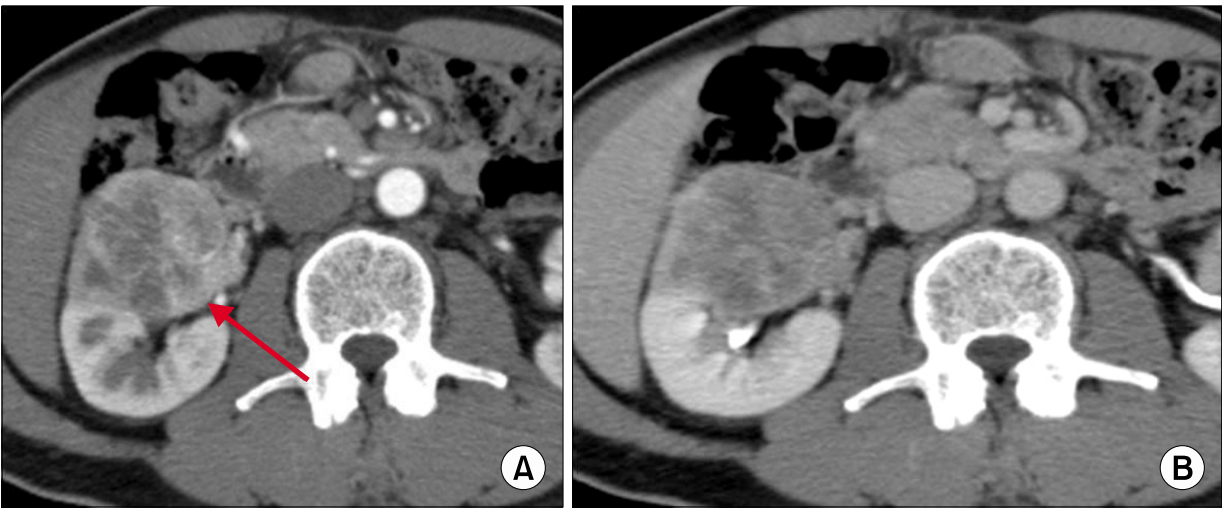

FiG. 2. Preoperative contrast-enhanced CT images of the right renal tumor. The tumor was heterogeneously enhanced, and the degree of enhancement was weaker than the normal renal parenchyma in the early phase (A). The tumor showed mild wash-out in the delayed phase (B). We also found right renal venous invasion (A). 


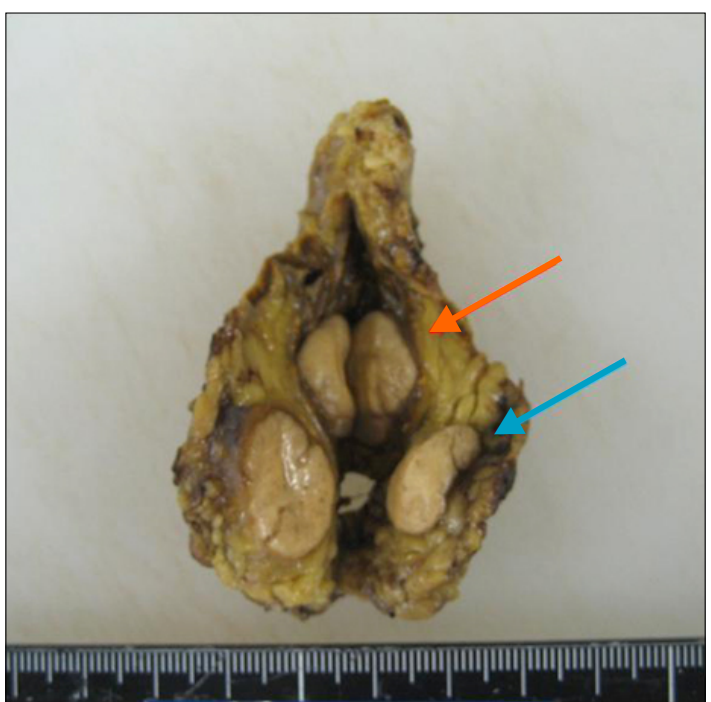

FiG. 3. Resected sample of the left adrenal gland (arrows: tumors). The tumors were macroscopically yellowish-white. Later, pathological examination revealed that the tumors were metastases of renal cell carcinoma.

adrenal metastasis occurred. Zornoza et al mentioned that the abundant blood flow and sinusoidal structure of the adrenal gland may affect this unique metastasis [10].

Concerning adrenal tumors, it is said that $50 \%$ to $75 \%$ of incidental adrenal tumors in oncology patients are metastases and $30 \%$ to $50 \%$ are nonfunctional adenomas. To distinguish a benign adenoma from a malignant adrenal mass, it is necessary to first identify the presence of fat in the tumor by nonenhanced CT or magnetic resonance imaging (in/opposite phase). Second, a CT protocol to evaluate the wash-out rate of contrast media in an adrenal mass may be useful.

In about one-third of RCC patients with isolated adrenal metastasis, surgical resection of the adrenal metastasis leads to an apparently curative outcome. In general, RCC patients with a solitary metastasis survive significantly longer than do those with multiple metastases. Complete resection of the primary renal and metastatic adrenal tumors should be the most important clinical treatment in cases such as this case.

To our knowledge, we are the first to report a case of RCC with double synchronous contralateral adrenal metastases. In cases of a unilateral renal tumor and contralateral adrenal tumor, this unique pathological state should be considered.

\section{Conflicts of Interest}

The authors have nothing to disclose.

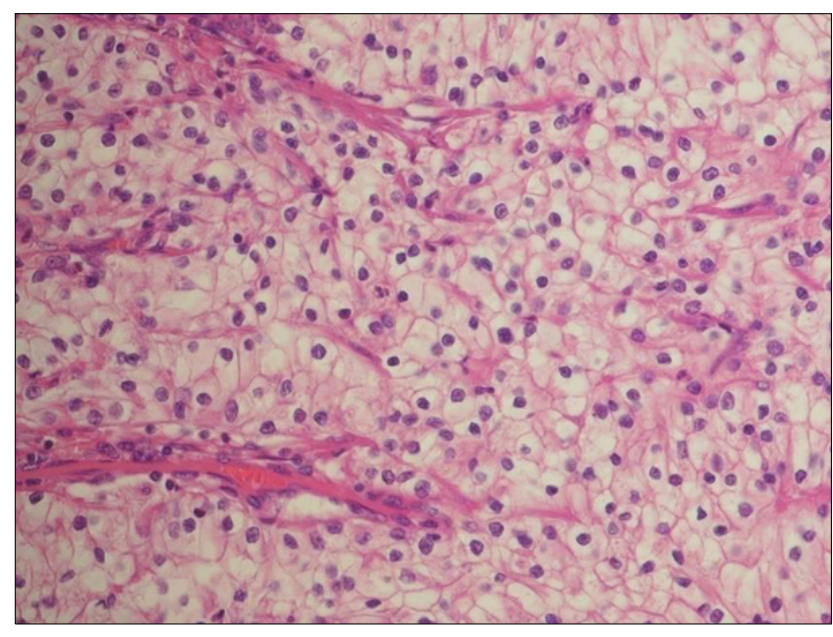

Fig. 4. Microscopic specimen of the right renal tumor. Pathological examination revealed that the tumor form was alveolar growth and there were many atypical cells that were composed of swelling nuclei in the clear cytoplasm. The tumor was diagnosed as clear cell-type renal cell carcinoma (H\&E, x100).

\section{REFERENCES}

1. Lau WK, Zinche H, Lohse CM, Cheville JC, Weaver AL, Blute ML. Contralateral adrenal metastasis of renal cell carcinoma: treatment, outcome and a review. BJU Int 2003;91:775-9.

2. Saitoh H, Nakayama M, Nakamura K, Satoh T. Distant metastasis of renal adenocarcinoma in nephrectomized cases. J Urol 1982;127:1092-5.

3. Sapienza P, Stipa F, Lucandri G, Baratti L, Delfino M, Mingazzini PL. Renal carcinoma with a solitary synchronous contralateral adrenal metastasis: a case report. Anticancer Res 1997;17:743-7.

4. Utsumi T, Suzuki H, Nakamura K, Kim W, Kamijima S, Awa Y, et al. Renal cell carcinoma with a huge solitary metastasis to the contralateral adrenal gland: a case report. Int J Urol 2008;15: 1077-9.

5. Abi-add AS, Belldegrum A, Dekernion JB. Renal cell carcinoma. Physiology, diagnosis, and therapy. World J Urol 1991;9:168-72.

6. Hellsten S, Berge T, Linell F. Clinically unrecognised renal cell carcinoma: aspects of tumor morphology, lymphatic and haematogenous metastatic spread. Br J Urol 1983;55:166-70.

7. Dieckmann KP, Wullbrand A, Krolzig G. Contralateral adrenal metastasis in renal cell cancer. Scand J Urol Nephrol 1996;30: $139-43$.

8. Robey EL, Schellhammer PF. The adrenal gland and renal cell carcinoma: Is ipsilateral adrenalectomy a necessary component of radical nephrectomy? J Urol 1986;135:453-5.

9. Angervall L, Wahlqvist L. Follow-up and prognosis of renal cell carcinoma in a series operated by perifascial nephrectomy combined with adrenalectomy and retroperitoneal lymphadenectomy. Eur Urol 1978;4:13-7.

10. Zornoza L, Bracken R, Wallace S. Radiologic features of adrenal metastases. Urology 1976;8:295-9. 